\title{
Are the spectra of geometrical operators in loop quantum gravity really discrete?
}

\author{
Bianca Dittrich ${ }^{1, a)}$ and Thomas Thiemann ${ }^{1,2, b)}$ \\ ${ }^{1}$ Perimeter Institute for Theoretical Physics, 31 Caroline Street North, \\ Waterloo, Ontario N2L 2Y5, Canada \\ ${ }^{2}$ MPIf. Gravitationsphysik, Albert-Einstein-Institut, Am Mühlenberg 1, \\ 14476 Potsdam, Germany
}

(Received 24 October 2007; accepted 30 November 2008; published online 15 January 2009)

\begin{abstract}
One of the celebrated results of loop quantum gravity (LQG) is the discreteness of the spectrum of geometrical operators such as length, area, and volume operators. This is an indication that the Planck scale geometry in LQG is discontinuous rather than smooth. However, there is no rigorous proof thereof at present. Because the aforementioned operators are not gauge invariant, they do not commute with the quantum constraints. The relational formalism in the incarnation of Rovelli's partial and complete observables provides a possible mechanism for turning a non-gaugeinvariant operator into a gauge invariant one. In this paper we investigate whether the spectrum of such a physical, that is, gauge invariant, observable can be predicted from the spectrum of the corresponding gauge variant observables. We will not do this in full LQG but rather consider much simpler examples where field theoretical complications are absent. We find, even in those simpler cases, that kinematical discreteness of the spectrum does not necessarily survive at the gauge invariant level. Whether or not this happens depends crucially on how the gauge invariant completion is performed. This indicates that "fundamental discreteness at the Planck scale in LQG" is far from established. To prove it, one must provide the detailed construction of gauge invariant versions of geometrical operators. (c) 2009 American Institute of Physics. [DOI: 10.1063/1.3054277]
\end{abstract}

\section{INTRODUCTION}

Among the candidates for a theory of quantum gravity, loop quantum gravity (LQG) (Ref. 1) has gained more and more popularity in recent years. One of the successes of LQG is the rigorous construction of (spatial) geometrical operators, such as the area, the volume, and the length operator, ${ }^{2,3}$ on the so-called kinematical Hilbert space of LQG. ${ }^{4}$ Moreover, it turns out that these geometrical operators have a discrete spectrum. In particular, there exists an area gap ${ }^{2,3}$ but apparently no volume gap (see Ref. 5). ${ }^{1}$

Another result is Ref. 6, which shows that the kinematical Hilbert space of LQG is the unique quantum representation for the holonomies and flux variables used in LQG satisfying certain covariance conditions with respect to the (spatial) diffeomorphism group. This shows that the discreteness of the spectra for the geometrical operators follows from a minimal set of requirements. Furthermore, the volume operator is pivotal to obtain an uv-finite quantization of the (gravity and matter) Hamiltonian constraints of the theory. ${ }^{7}$

\footnotetext{
${ }^{a)}$ Electronic mail: bdittrich@perimeterinstitute.ca.

${ }^{b}$ Electronic mail: thomas.thiemann@aei.mpg.de.

${ }^{1}$ There exist a volume gap on the subspace of the kinematical Hilbert space spanned by spin networks with vertex valence less than or equal to 4. However, on the full kinematical Hilbert space numerical evidence for an accumulation point at zero was found, see the last three references in Ref. 5.
} 
In summary, the geometrical operators play a very important role for the structure and further development of LQG. However, so far the discreteness of their spectrum is a result at the kinematical level only. By "kinematical level" we mean that the geometrical operators defined so far are gauge dependent, i.e., they are not invariant under space-time diffeomorphisms. True observables have to be gauge independent. In the canonical formalism such observables are known as Dirac observables. Physical measurements are described by Dirac observables and not by kinematical observables.

The explicit construction of Dirac observables in general relativity is very difficult since it requires the solution of the dynamics of the theory. However, methods to obtain Dirac observables (at least as classical phase space functions) are available. ${ }^{8,9}$ With these methods one can construct the so-called complete observables, ${ }^{10}$ a specific type of relational observables. The main idea here is to use some sets of fields $T$ as "clocks and rods" and to express another field $f$ (the "partial observable") in the coordinates defined by these clocks and rods. Under a diffeomorphism both $f$ and $T$ transform in the same way, so that the relation between $f$ and $T$ does not change. More details and a proof that the resulting phase space functions are indeed Dirac observables can be found in the second reference in Ref. 8.

Indeed, one way to construct Dirac observables out of the geometrical operators mentioned above is to use matter fields in order to localize the region which one wants to measure, for instance, the (spatial or space-time) volume. This is also suggested in Ref. 2. The question would be whether or not the Dirac observables constructed in this manner (assuming that such Dirac observables can be quantized as self-adjoint operators on a yet to be constructed physical Hilbert space) have a discrete spectrum. Reference 2 argues for a discrete spectrum of the Dirac observables.

Contrary to this expectation, we will show that in this note, without further assumptions, it is impossible to make any predictions about the spectra of the complete observables, even if the spectra of the partial observables and the clocks are known. We will do that by means of quite simple examples with finitely many degrees of freedom, where the physical Hilbert space can be explicitly constructed. In more complicated (field theory) examples, even the physical Hilbert space might not be unique and the spectra of observables could, in principle, depend on the choice of the Hilbert space.

The plan of the paper is as follows: In Sec. II we will explain the method of partial and complete observables for systems with one constraint. This allows us to associate with a gauge variant function (the partial observable) a one-parameter family of gauge invariant functions (the complete observable). Furthermore, one has to choose a clock. We can then consider in what way the spectrum of the complete observables depends on the spectrum of the partial observable, the clock, and the constraint. In Sec. III we will show with the help of concrete examples that every possible combination of continuous and discrete spectra for the constraint, the partial observable, the clock, and the complete observable can be realized. We will end with a discussion of the results and their meaning for LQG. In particular, we will comment on earlier results within the LQG literature on the physical spectrum of geometrical operators in the context of $2+1$ Lorentzian gravity $^{11,12}$ and $3+1$ Lorentz covariant gravity. ${ }^{13}$

In Appendix B we consider an additional toy model which is geared to constructing a gauge invariant observable from a baby version of a volume operator by means of a baby version of a scalar field as discussed in Ref. 2. We find that in contrast to the arguments spelled out there, the kinematically discrete spectrum switches to a gauge invariant continuous one.

\section{PARTIAL AND COMPLETE OBSERVABLES}

Here we will explain the method of partial and complete observables. The examples we are going to discuss exhibit only one constraint, i.e., only one gauge degree of freedom. We will comment on the case with several constraints (general relativity has infinitely many constraints) later on.

Let us start with an example, namely, the relativistic particle in two space-time dimensions. Here we have the mass shell constraint 


$$
C=p_{0}^{2}-p_{1}^{2}-m^{2}
$$

where $m$ is the mass and $\left(p_{0}, p_{1}\right)$ the momentum of the particle. Now we have to choose a clock variable $T$ and another partial observable $f$. Our choice is $T=x_{0}$ and $f=x_{0} p_{1}-x_{1} p_{0}$, where $\left(x_{0}, x_{1}\right)$ is the position vector of the particle. The complete observable $F(\tau)$, where $\tau$ is a phase space independent parameter, is defined to be the gauge invariant phase space function with the following property: On the hypersurface $T=\tau$ it should coincide with the phase space function $f$. Gauge invariance means here that $F(\tau)$ has to be invariant under the flow of the mass shell constraint (2.1), i.e., that $\{F(\tau), C\}=0$, where the Poisson brackets are defined by $\left\{x_{i}, p_{j}\right\}=\delta_{i j}$.

One way to find the complete observable is to compute the flow of the clock variable $T$ under the constraint $C$, that is,

$$
T(t):=\sum_{k=0}^{\infty} \frac{t^{k}}{k !}\{T, C\}_{k},
$$

where $\{g, C\}_{k+1}=\left\{\{g, C\}_{k}, C\right\},\{g, C\}_{0}=g$ are iterated Poisson brackets. Next, one has to solve the equation $T(t) \stackrel{!}{=} \tau$ for $t$. The solution $t_{s}=t_{s}(\tau)$ is, in general, also phase space dependent. For the relativistic particle we have

$$
T(t)=x_{0}+2 t p_{0} \stackrel{!}{=} \tau
$$

Hence, $t_{s}=\frac{1}{2} p_{0}^{-1}\left(\tau-x_{0}\right)$.

Now we have to insert the solution $t_{s}$ into the flow of $f$ under the constraint $C$. This gives the complete observable $F(\tau)$,

$$
F(\tau)=f\left(t_{s}(\tau)\right)=\sum_{k=0}^{\infty} \frac{\left(t_{s}(\tau)\right)^{k}}{k !}\{f, C\}_{k} .
$$

Since for the relativistic particle $f(t)=\left(x_{0}+2 t p_{0}\right) p_{1}-\left(x_{1}-2 t p_{1}\right) p_{0}$, we find

$$
F(\tau)=\tau p_{1}-\left(x_{1} p_{0}-\left(\tau-x_{0}\right) p_{1}\right)=2 \tau p_{1}-\left(x_{0} p_{1}+x_{1} p_{0}\right) .
$$

Indeed (2.5) coincides with $f$ on the hypersurface $T=x^{0}=\tau$ and is invariant under the flow of $C$.

This construction can be generalized to systems with arbitrary many (first class) constraints by introducing as many clock variables $T_{i}$ as there are constraints $C_{i}$, where $i$ is in some index set $I$. The complete observable $F\left(\tau_{i}\right)$ associated with these clock variables and a partial observable $f$ is defined to be the gauge invariant function which coincides on the hypersurface $\left\{T_{i}=\tau_{i} \forall i \in I\right\}$ with the function $f$. For methods to compute complete observables see Ref. 8.

We want to remark that the complete observable might not always be well defined. For instance, the surface $T=\tau$ might meet a gauge orbit several times which leads to multiple solutions of the equation $T(t) \stackrel{!}{=} \tau$ and hence might lead to multiple valued complete observables as is discussed in the first reference in Ref. 8. Some examples in the next section will have multiple solutions $t_{s}$ of the equation $T(t) \stackrel{!}{=} \tau$ but in these the partial observable $f$ is chosen such that $f(t)$ coincides on all solutions $t_{s}$ and hence we will always get a well defined complete observable.

On the other hand, it might happen that for a fixed parameter $\tau$ the surface $T=\tau$ does not meet a certain set of gauge orbits at all. Here we will assume that the parameter $\tau$ is always chosen such that one can define the complete observable at least on an open set of the phase space. In our examples this complete observable can always be continued analytically to a gauge invariant function defined on the whole phase space. ${ }^{2}$

\footnotetext{
${ }^{2}$ This convention is necessary, for instance, for the examples with the rotation generator as constraint. Note that with this convention it may happen that the complete observable $F(\tau)$ assumes negative values whereas the partial observable $f$ is non-negative.
} 
Furthermore, often we will give the complete observables modulo terms that vanish on the constraint hypersurface, since these terms are not relevant for the quantization of the observables on the physical Hilbert space.

With the relativistic particle we already have an example where the spectrum of the quantization of the partial observable $f=x_{0} p_{1}-x_{1} p_{0}$ is discrete [since it generates rotations in the $\left(x_{0}, x_{1}\right)$ plane] and the spectrum of the quantization of the corresponding complete observable $F(\tau=0)=$ $-x_{0} p_{1}-x_{1} p_{0}$ is continuous (since it generates boosts).

Such a change of the spectral properties can be understood in the following way: Heuristically ${ }^{3}$ the quantization $\hat{f}$ of a (smooth) phase space function $f$ has a discrete spectrum or a continuous spectrum if the vector field $\chi_{f}$ associated with the phase space function $f$ generates compact orbits or noncompact orbits, respectively.

The vector field $\chi_{f}$ associated with $f$ is defined by the equation

$$
\{g, f\}=\chi_{f}(g),
$$

which has to hold for every smooth function $g$. Here the Poisson brackets $\{\cdot, \cdot\}$ are defined via the symplectic form $\omega$ defined on the phase space in question.

Assume that the equation $T=\tau$ defines a good gauge fixing, i.e., that the surface $T=\tau$ meets each gauge orbit once and only once. As it is explained in Refs. 8 and 9 in this case the following holds: The space of all complete observables with this choice of clock variable $T$ can be mapped via a symplectomorphism onto the reduced phase space obtained with the gauge fixing $T=\tau$ and equipped with the Dirac bracket $\{\cdot,\}^{D}$. This map is given by mapping the complete observable associated with $f$ to the gauge restriction of $f$, i.e., the restriction of the function $f$ to the reduced phase space $\{C=0, T=\tau\}$.

Hence, considering complete observables and the symplectic flows they generate is equivalent to considering the reduced phase space $\{C=0, T=\tau\}$ with its induced symplectic form, which is given by the Dirac bracket. That is, a complete observable associated with a function $f$ generates compact orbits if and only if this is the case for the symplectic flow of the gauge restriction of $f$ defined via the Dirac bracket. This symplectic flow preserves, in particular, the reduced phase space $\{C=0, T=\tau\}$. In fact, the vector fields $\chi_{f}^{D}$ associated with the gauge restriction of $f$ via the Dirac bracket can be understood as the vector fields $\chi_{f}$ projected to the hypersurface $\{C=0, T$ $=\tau\}$ : since the flow defined via the Dirac bracket does not leave the hypersurface $\{C=0, T=\tau\}$, the associated vector field $\chi_{f}^{D}$ has to be tangent to the hypersurface.

Now to determine whether the orbits generated by the vector field $\chi_{f}$ are compact or not cannot, in general, be concluded from local considerations only. It might change under the projection ${ }^{4}$ onto the reduced phase space. Also, the global properties of the reduced phase space and how the reduced phase space is embedded into the full phase space are important. This explains the different spectra for the partial observable $f$ and the complete observable $F(\tau)$. Moreover, this argument shows that it is very difficult to predict which kind of spectrum the complete observables will have, since it requires control of the global features of the reduced phase space.

In Sec. III we will give examples for all possible combinations of discrete (i.e., pure point) or continuous (i.e., absolutely continuous) spectra for the (quantized) constraint, the clock variable $T$, the partial observable $f$, and the complete observable $F(\tau)$.

\section{THE EXAMPLES}

In our examples we will use the phase space $\mathbb{R}^{2} \times \mathbb{R}^{2}$ with canonical coordinates $\left(x_{1}, p_{1}, x_{2}, p_{2}\right)$ and Poisson brackets $\left\{x_{i}, p_{j}\right\}=\delta_{i j}$ (where $\delta_{i j}$ is the Kronecker symbol).

\footnotetext{
${ }^{3}$ For instance, the Bohr-Sommerfeld quantization rule (Ref. 14) applicable to systems with periodic motion is a heuristic method which connects the energy gaps to properties of the phase space orbits as generated by the Hamiltonian.

${ }^{4}$ Note that not the orbits themselves are projected but rather the Hamiltonian vector fields. Hence compact orbits may change to noncompact ones under the projection of the generating vector fields.
} 
We choose the space of square integrable functions $L_{2}\left(\mathbb{R}^{2}\right)$ as the kinematical Hilbert space. The quantized configuration coordinate functions $\hat{x}_{j}$ act as multiplication operators $\hat{x}_{j}=x_{j}$ and the quantized momenta as derivative operators $\hat{p}_{j}=-i \hbar \partial_{j}$.

We will start our considerations with the constraint $\hat{C}_{d}=\hat{x}_{1} \hat{p}_{2}-\hat{x}_{2} \hat{p}_{1}$, which is the generator of rotations on $\mathbb{R}^{2}$. The constraint operator has a discrete spectrum $\{\hbar z, z \in \mathbb{Z}\}$ (indicated by the index d). We will assume that the partial and complete observables have either an entirely discrete (i.e., pure point) spectrum or an entirely continuous (i.e., absolutely continuous) spectrum and that the spectral type of the complete observable does not depend on the particular value of the parameter $\tau$. Then there exist eight combinations for the spectra of the clock variable $\hat{T}$, the partial observable $\hat{f}$, and the complete observable $\hat{F}(\tau)$. We will indicate these combinations by $(d, i, j, k)$ with $i, j, k \in\{c, d\}$ for the spectrum $i$ of the clock variable $\hat{T}$, the spectrum $j$ of the partial observable $\hat{f}$, and the spectrum $k$ of the complete observable $\hat{F}(\tau)$.

The construction of the physical Hilbert space for the constraint $\hat{C}_{d}$ is straightforward. The constraint has a pure point spectrum and moreover the point zero is included in this spectrum so that we have solutions $\psi_{\text {phys }}$ to the constraint equation $\hat{C}_{d} \psi_{\text {phys }}=0$ that are elements of the kinematical Hilbert space. A physical inner product is given by the restriction of the kinematical inner product to these solutions.

The gauge invariant functions we will encounter in our examples of complete observables are given by $r^{2}:=x_{1}^{2}+x_{2}^{2}$ and $h:=x_{1}^{2}+x_{2}^{2}+p_{1}^{2}+p_{2}^{2}$. The corresponding quantizations have a continuous spectrum and a discrete spectrum, respectively. Details on the physical Hilbert space and the algebra of Dirac observables can be found in Appendix A.

We will begin the discussion of the examples with the case $(d, d, d, d)$. Here we choose as clock variable and as partial observable

$$
T=x_{1}^{2}+p_{1}^{2}, \quad f=x_{2}^{2}+p_{2}^{2},
$$

which are the harmonic oscillators in the $x_{1}$ and $x_{2}$ coordinates, respectively. The corresponding quantum operators have obviously a discrete spectrum.

One way to find $^{5}$ the complete observable is to first solve the equation

$$
T(t):=\sum_{k=0}^{\infty} \frac{t^{k}}{k !}\left\{T, C_{d\}} \stackrel{!}{=}^{=} \tau\right.
$$

for $t$ and then to insert the solutions of this equation into $f(t)$. However, there is a shorter way: Note that

$$
T(t)=x_{1}^{2} \cos ^{2}(t)+x_{2}^{2} \sin ^{2}(t)-2 x_{1} x_{2} \cos (t) \sin (t)+p_{1}^{2} \cos ^{2}(t)+p_{2}^{2} \sin ^{2}(t)-2 p_{1} p_{2} \cos (t) \sin (t) .
$$

Hence, we have for solutions $t_{s}$ of the equation $T(t)=\tau$

$$
2 x_{1} x_{2} \cos \left(t_{s}\right) \sin \left(t_{s}\right)+2 p_{1} p_{2} \cos \left(t_{s}\right) \sin \left(t_{s}\right)=x_{1}^{2} \cos ^{2}\left(t_{s}\right)+x_{2}^{2} \sin ^{2}\left(t_{s}\right)+p_{1}^{2} \cos ^{2}\left(t_{s}\right)+p_{2}^{2} \sin ^{2}\left(t_{s}\right)-\tau \text {. }
$$

We use this equation in

\footnotetext{
${ }^{5}$ The following exceptionally simple examples were actually not found by using the partial observable method directly but by splitting an observable under the respective symmetry into two nonobservables which are then the two candidate partial observables. This works because in most of our elementary examples the complete observables are a linear combination of the partial observable and the clock variable. As has been pointed out by Christian Fleischhack, this method can of course be used in principle in a wider context.
} 


$$
\begin{aligned}
F(\tau)= & f\left(t_{s}\right)=2 x_{1} x_{2} \cos \left(t_{s}\right) \sin \left(t_{s}\right)+2 p_{1} p_{2} \cos \left(t_{s}\right) \sin \left(t_{s}\right)+x_{2}^{2} \cos ^{2}\left(t_{s}\right)+x_{1}^{2} \sin ^{2}\left(t_{s}\right)+p_{2}^{2} \cos ^{2}\left(t_{s}\right) \\
& +p_{1}^{2} \sin ^{2}\left(t_{s}\right)
\end{aligned}
$$

and find

$$
F(\tau)=x_{1}^{2}+x_{2}^{2}+p_{1}^{2}+p_{2}^{2}-\tau,
$$

which is the sum of two harmonic oscillator Hamiltonians minus a constant $\tau$. The corresponding quantum operator has a discrete spectrum.

Hence, we found an example for the case $(d, d, d, d)$. Examples for all the other cases $(d, i, j, k)$ can be found in a similar manner. The examples are summarized below:

\begin{tabular}{c|ccc} 
Case & $T$ & $f$ & $F(\tau)$ \\
\hline$(d, d, d, d)$ & $x_{1}^{2}+p_{1}^{2}$ & $x_{2}^{2}+p_{2}^{2}$ & $x_{1}^{2}+p_{1}^{2}+x_{2}^{2}+p_{2}^{2}-\tau$ \\
$(d, c, d, d)$ & $\left(1-\omega^{2}\right) x_{1}^{2}+$ & $p_{1}^{2}+\omega^{2} x_{1}^{2}+$ & $x_{1}^{2}+p_{1}^{2}+x_{2}^{2}+p_{2}^{2}-\tau$ \\
& $\left(1-\omega^{\prime 2}\right) x_{2}^{2}$ & $p_{2}^{2}+\omega^{\prime 2} x_{2}^{2}$ & \\
$(d, d, c, d)$ & $p_{1}^{2}+\omega^{2} x_{1}^{2}+$ & $\left(1-\omega^{2}\right) x_{1}^{2}+$ & $x_{1}^{2}+p_{1}^{2}+x_{2}^{2}+p_{2}^{2}-\tau$ \\
& $p_{2}^{2}+\omega^{\prime 2} x_{2}^{2}$ & $\left(1-\omega^{\prime 2}\right) x_{2}^{2}$ & \\
$(d, c, c, d)$ & $x_{1}^{2}+p_{2}^{2}$ & $x_{2}^{2}+p_{1}^{2}$ & $x_{1}^{2}+p_{1}^{2}+x_{2}^{2}+p_{2}^{2}-\tau$ \\
$(d, d, d, c)$ & $\left(p_{1}^{2}+\omega^{2} x_{1}^{2}\right)-$ & $-\left(p_{1}^{2}+\omega^{\prime 2} x_{1}^{2}\right)+$ & $\left(\omega^{2}-\omega^{\prime 2}\right)\left(x_{1}^{2}+x_{2}^{2}\right)-\tau$ \\
& $\left(p_{2}^{2}+\omega^{\prime 2} x_{2}^{2}\right)$ & $\left(p_{2}^{2}+\omega^{2} x_{2}^{2}\right)$ & \\
$(d, d, c, c)$ & $p_{1}^{2}+x_{1}^{2}$ & $p_{1}^{2}-x_{2}^{2}$ & $-x_{1}^{2}-x_{2}^{2}+\tau$ \\
$(d, c, d, c)$ & $p_{1}^{2}-x_{2}^{2}$ & $p_{1}^{2}+x_{1}^{2}$ & $x_{1}^{2}+x_{2}^{2}+\tau$ \\
$(d, c, c, c)$ & $x_{1}^{2}$ & $x_{2}^{2}$ & $x_{1}^{2}+x_{2}^{2}-\tau$
\end{tabular}

We will now turn to a constraint with a continuous spectrum. We use the same phase space and the same kinematical Hilbert space as before, namely, $\mathbb{R}^{2} \times \mathbb{R}^{2}$ and $L_{2}\left(\mathbb{R}^{2}\right)$, respectively. We will work with the momentum constraint $C_{c}=p_{1}$, whose quantization $\hat{C}_{c}=-i \hbar \partial_{1}$ has a continuous spectrum.

The Dirac observable algebra for this constraint is spanned by $x_{2}$ and $p_{2}$, i.e., it is given by phase space functions which do not depend on $x_{1}$ or $p_{1}$. Physical wave functions are wave functions which do not depend on $x_{1}$. A physical inner product can be defined by omitting the integration over $x_{2}$ in the kinematical inner product. Hence, we are left with a physical Hilbert space $L_{2}\left(\mathbb{R}, d x_{2}\right)$ which carries the usual representation of the basic Dirac observables $\hat{x}_{2}$ and $\hat{p}_{2}$.

The calculation of the complete observables is straightforward. In table (3.8) we have listed the examples for all possible cases $(c, i, j, k)$ where again $i, j, k$ can take values $d$ or $c$ and indicate a discrete or continuous spectrum, respectively, of the corresponding quantum observable. Note that for the case $(c, d, d, d)$ we make use of the fact that two observables are equivalent if they differ by a term vanishing on the constraint surface,

\begin{tabular}{c|ccc} 
Case & $T$ & $f$ & $F(\tau)$ \\
\hline$(c, d, d, d)$ & $x_{1}^{2}+p_{1}^{2}$ & $x_{1}^{2}+p_{1}^{2}+x_{2}^{2}+p_{2}^{2}$ & $x_{2}^{2}+p_{2}^{2}+\tau$ \\
$(c, c, d, d)$ & $x_{1}^{2}$ & $x_{1}^{2}+p_{1}^{2}+x_{2}^{2}+p_{2}^{2}$ & $x_{2}^{2}+p_{2}^{2}+\tau$ \\
$(c, d, c, d)$ & $x_{1}^{2}+p_{1}^{2}+x_{2}^{2}+p_{2}^{2}$ & $x_{1}^{2}$ & $-x_{2}^{2}-p_{2}^{2}+\tau$ \\
$(c, c, c, d)$ & $x_{1}+p_{2}^{2}$ & $x_{1}-x_{2}^{2}$ & $-x_{2}^{2}-p_{2}^{2}+\tau$ \\
$(c, d, d, c)$ & $x_{1}^{2}+p_{1}^{2}$ & $\left(x_{1} p_{2}-x_{2} p_{1}\right)^{2}$ & $\tau p_{2}^{2}$ \\
$(c, d, c, c)$ & $x_{1}^{2}+p_{1}^{2}+x_{2}^{2}+p_{2}^{2}$ & $x_{1}^{2}+x_{2}^{2}$ & $-p_{2}^{2}+\tau$ \\
$(c, c, d, c)$ & $x_{1}^{2}+x_{2}^{2}$ & $x_{1}^{2}+p_{1}^{2}+x_{2}^{2}+p_{2}^{2}$ & $p_{2}^{2}+\tau$ \\
$(c, c, c, c)$ & $x_{1}$ & $x_{1}+x_{2}$ & $x_{2}+\tau$
\end{tabular}


Consider. in particular. the cases $(c, c, d, d)$ and $(c, c, d, c)$ which can be generalized to

$$
\begin{gathered}
T=x_{1}^{2}+\gamma x_{2}^{2}, \\
f=x_{1}^{2}+p_{1}^{2}+x_{2}^{2}+p_{2}^{2}, \\
F(\tau) \simeq(1-\gamma) x_{2}^{2}+p_{2}^{2}+\tau,
\end{gathered}
$$

with $0 \leq \gamma<1$ or $\gamma \geq 1$ for a discrete spectrum of the complete observable or a continuous spectrum of the complete observable, respectively. The symbol $\simeq$ indicates that the last equation in (3.9) holds modulo terms which vanish on the constraint hypersurface. Hence, we have an example where just changing the clock variable $T$ leads to the change of the spectrum of the complete observable.

Examples with more than one constraint can be easily constructed by taking "a direct sum" of the above examples for one constraint: For instance, consider the constraints $C_{1}=p_{1}$ and $C_{2}=p_{3}$ on a phase space $\mathbb{R}^{4} \times \mathbb{R}^{4}$. Choose as partial observables

$$
\begin{gathered}
T_{1}=x_{1}^{2}, \\
T_{2}=x_{3}^{2}, \\
f=x_{1}^{2}+p_{1}^{2}+x_{2}^{2}+p_{2}^{2}+x_{3}^{2}+p_{3}^{2}+x_{4}^{2}+p_{4}^{2} .
\end{gathered}
$$

The corresponding complete observable is $F\left(\tau_{1}, \tau_{2}\right) \simeq x_{2}^{2}+p_{2}^{2}+x_{4}^{2}+p_{4}^{2}+\tau_{1}+\tau_{2}$. Hence, we have an example with two constraints and two clock variables with a continuous spectrum and a partial observable $f$ and a complete observable $F\left(\tau_{1}, \tau_{2}\right)$ with a discrete spectrum.

\section{DISCUSSION}

The examples show that it is very difficult to make any predictions about the spectra of physical observables even if the spectra of gauge variant observables are known to which the physical observables are associated in a certain sense (e.g., here through the method of partial and complete observables). The examples considered here are very simple and do not include further complications one would expect for the geometrical operators in LQG. For instance, one might consider space-time geometrical operators rather than spatial ones.

Among earlier work on these issues we select the following three: In Ref. 11 the authors consider $2+1$ Lorentzian gravity without matter and construct a length operator within the Hamiltonian theory. Since the authors do not require that the spatial slices be spacelike, the length operator measures the length of timelike or spacelike curves. The spacelike spectrum is continuous while the timelike one is discrete, corresponding to the coexistence of a continuous and a discrete spectrum of the Casimir for $\mathrm{SO}(1,2)$. However, the spectrum is at the kinematical level only and it is unknown what happens when one implements the dynamics. Of more relevance to our paper is Ref. 12 which concerns Riemannian $2+1$ gravity with point particles. Now the length operator can be made into a Dirac observable and the spectrum is discrete for both the kinematical and the physical length observable! However, it is not clear whether a similar construction will also work in $3+1$ dimensions, and the analysis is done for the Riemannian case only. Finally there is a body of literature called "Lorentz covariant gravity" in $3+1$ dimensions, see, for instance, Ref. 13, where one keeps the connection a Lorentz connection at the price of the connections not to Poisson commute. Hence, one does not have any (connection) representation and thus rigorous spectral theory cannot be performed.

Our heuristic argument of how one could understand the change from discrete to continuous spectra and vice versa shows that the global features of the kinematical and reduced phase space 
play an important role. However, it is very hard to get complete control over the reduced phase space of general relativity. It might be therefore complicated to give any conditions that would ensure the discreteness of the spectra for the complete observables.

As a more general lesson these examples show that physical observables might have very unexpected properties. Here we considered the spectra of physical observables. Other properties are, for instance, commutation relations of physical observables; see, for instance, the third reference in Ref. 8. Moreover, there are indications that considering physical observables might lead to a notion of noncommutative space-time. ${ }^{15}$

Although we cannot say yet whether or not the true physical geometrical operators of LQG will have discrete spectra without spelling out additional details, we want to emphasize that their kinematical versions are still important. For instance, the kinematical volume operator enters the definition of the Hamiltonian constraint and surely many other Dirac observables which are gauge invariant aggregates built out of gauge variant operators.

\section{ACKNOWLEDGMENTS}

It is a pleasure to thank Christian Fleischhack for fruitful discussions. Research at the Perimeter Institute for Theoretical Physics is supported in part by the Government of Canada through NSERC and by the Province of Ontario through MRI.

\section{APPENDIX A: EXAMPLE, PHYSICAL HILBERT SPACE}

Here we will briefly discuss the representation of the Dirac observable algebra on the physical Hilbert space for the constraint $\hat{C}_{d}=\hat{x}_{1} \hat{p}_{2}-\hat{x}_{2} \hat{p}_{1}$. The Dirac observable algebra is spanned by

$$
\hat{d}=\frac{1}{2}\left(\hat{x}_{i} \hat{p}_{i}+\hat{p}_{i} \hat{x}_{i}\right)=\hat{x}_{i} \hat{p}_{i}-i \hbar, \quad \hat{e}^{+}=\hat{x}_{i} \hat{x}_{i}, \quad \hat{e}^{-}=\hat{p}_{i} \hat{p}_{i},
$$

where we sum over repeated indices. With this operator ordering for $\hat{d}$ the observables (A1) represent the $\operatorname{sl}(2, \mathbb{R})$ algebra:

$$
\left[\hat{d}, \hat{e}^{ \pm}\right]=\mp 2 i \hbar e^{ \pm}, \quad\left[\hat{e}^{+}, \hat{e}^{-}\right]=4 i \hbar \hat{d} .
$$

The constraint has a pure point spectrum and, moreover, the point zero is included in this spectrum so that we have solutions $\psi_{\text {phys }}$ to the constraint equation $\hat{C}_{d} \psi_{\text {phys }}=0$ that are elements of the kinematical Hilbert space. A physical inner product is given by the restriction of the kinematical inner product to these solutions. Explicitly we can change to polar coordinates $r \in \mathbb{R}_{+}, \theta$ $\in[0,2 \pi)$ :

$$
x_{1}=r \cos \theta \quad x_{2}=r \sin \theta,
$$

so that the constraint operator $\hat{C}_{d}$ and the Dirac observable algebra (A1) become

$$
\begin{gathered}
\hat{C}_{d}=-i \hbar\left(x_{1} \partial_{2}-x_{2} \partial_{1}\right)=-i \hbar \partial_{\theta}, \\
\hat{d}=-i \hbar\left(x_{1} \partial_{1}+x_{2} \partial_{2}+1\right)=-i \hbar\left(r \partial_{r}+1\right), \\
\hat{e}^{+}=\left(x_{1}^{2}+x_{2}^{2}\right)=r^{2}, \\
\hat{e}^{-}=-\hbar^{2}\left(\partial_{1}^{2}+\partial_{2}^{2}\right)=-\hbar^{2}\left(\partial_{r}^{2}+r^{-1} \partial_{r}+r^{-2} \partial_{\theta}^{2}\right)
\end{gathered}
$$

on the kinematical Hilbert space $L_{2}\left(r d r d \theta, \mathbb{R}_{+} \times[0,2 \pi)\right)$. The physical states are the states which do not depend on the angular coordinate $\theta$ and the physical inner product can be written as 


$$
\left(\psi_{\text {phys }}, \phi_{\text {phys }}\right)_{\text {phys }}=\int_{\mathrm{R}_{+}} \overline{\psi_{\text {phys }}(r)} \phi_{\text {phys }}(r) r d r .
$$

The representation (A4) of the Dirac observable algebra (A1) is unitary and irreducible. It is equivalent to the representation $D_{1 / 2}^{+}$(see, for instance, Ref. 16) from the discrete series of representations of $\operatorname{sl}(2, \mathbb{R})$. In this representation the operators $\hat{e}^{+}$and $\hat{e}^{+}+\hat{e}^{-}$(which is the twodimensional harmonic oscillator restricted to states with vanishing angular momentum) have an absolutely continuous spectrum and a pure point spectrum, respectively. These Dirac observables appear as complete observables in our examples.

\section{APPENDIX B: BABY VERSION OF LQG GEOMETRICAL OPERATORS}

The examples considered so far could be taken as irrelevant for the situation in LQG for two reasons: First, the configuration space of the models was noncompact while for the geometry part of LQG the configuration space was compact. Second, in all the examples considered it so happened that $f, T$ did not Poisson commute whenever the spectrum changed. However, the idea of Ref. 2 was to use for $T$ scalar matter while $f$ is a geometry function; hence $f, T$ did Poisson commute there.

In Ref. 17 we find an example based on $\operatorname{si}(2, R)$ where a continuous kinematical spectrum switches to a discrete gauge invariant one. However, there the configuration space is still noncompact and, furthermore, the switch happens in the wrong direction. Thus, we now explicitly display an example where both of the above-mentioned properties that are missing in our models are satisfied and still the switch occurs.

Consider the phase space $T^{*}\left(S^{1} \times R\right)$ of a particle moving on a cylinder. Denote by $A$ the angle configuration variable ("connection") and by $E$ its conjugate momentum ("flux"). Recall that all geometrical operators of LQG are compound operators built from fluxes and they inherit their spectral discreteness from that of the flux operators. Denote by $\Phi$ the axis configuration variable ("scalar field") and by $\Pi$ its conjugate momentum. Of course, $A$ is not a globally defined function and we must switch to the "holonomy" $h=\exp (i A)$. is,

We impose as constraint that the particle must spiral around the cylinder with period $2 \pi$; that

$$
C=A-\left(\Phi-2 \pi\left[\frac{\Phi}{2 \pi}\right]\right),
$$

where [.] denotes the Gauss bracket. In the form (B1) the constraint is not differentiable; hence, we pass to the equivalent version

$$
C=\exp (i[A-\Phi])-1=0 .
$$

In this form the constraint is not real valued but that will not pose any problems. [Alternatively work with the reducible system $C_{1}=\cos (A-\Phi)-1$ and $C_{2}=\sin (A-\Phi)$. The constraint $C_{2}$ is used to determine the gauge flow on the constraint hypersurface defined by (B1), whereas $C_{1}$ is only needed to exclude the points with $A-\Phi=\pi$ and so on from consideration. One cannot reduce this system to only one real and differentiable constraint: this would require a continuous and periodic function that is (a) vanishing at $2 \pi z, z \in \mathbb{Z}$, and only at these points and (b) has nonvanishing and well defined derivative at these points. A continuous function vanishing at, say, 0 and $2 \pi$ and having the same derivative different from zero at these points has to vanish at least at one point in the open interval $(0,2 \pi)$.]

As clock we choose $T=\Pi$ and as partial observable $f=E$. A straightforward calculation reveals that the corresponding complete observable is given by

$$
F=E+\Pi-\tau,
$$

which is essentially the total momentum of the particle. 
The kinematical Hilbert space is given by $\mathcal{H}_{\text {kin }}=L_{2}\left(S^{1}, d A / 2 \pi\right) \otimes L_{2}(R, d \Phi)$. Operators are represented as $\hat{\Pi}=i d / d \Phi, \hat{E}=i d / d A=-h d / d h$ while $\Phi, A$ (or rather $h$ ) become multiplication operators. Clearly, $\hat{\Pi}, \hat{E}, \hat{\Phi}$ are self-adjoint while $\hat{h}$ is unitary. The spectrum of $\hat{E}$ is discrete and takes integer values with eigenfunctions given by $T_{n}=h^{n}$ ("spin network functions").

To compute the physical Hilbert space we define the rigging map ${ }^{6}$

$$
\eta(\psi):=\delta(\hat{C}) \psi
$$

The $\delta$ distribution is given by

$$
\delta(\hat{C})=\delta_{R}\left(A-\left(\Phi-2 \pi\left[\frac{\Phi}{2 \pi}\right]\right)\right)=\sum_{n} \delta_{R}(\Phi, A+2 \pi n)=\sum_{n} \exp (i n[A-\Phi])=\delta_{S^{1}}(A-\Phi),
$$

where in the last step we have applied a Poisson resummation. The physical inner product on the solutions $\eta(\psi)$ is given by

$$
\left\langle\eta(\psi), \eta\left(\psi^{\prime}\right)\right\rangle_{\mathrm{phys}}=\left\langle\psi, \delta(\hat{C}) \psi^{\prime}\right\rangle_{\mathrm{kin}}=\left\langle\tilde{\psi}, \tilde{\psi}^{\prime}\right\rangle_{L_{2}(R, d \Phi)},
$$

where $\tilde{\psi}(\Phi)=\psi(A=\Phi, \Phi)$ which is well defined because $\psi$ is periodic in $A$.

One can also get this by making the Fourier expansion

$$
\psi(A, \Phi)=\sum_{n} T_{n}(A) \psi_{n}(\Phi)
$$

and solve $\exp (i[\hat{A}-\hat{\Phi}]) \psi=\psi$ for the coefficients $\psi_{n}$.

It is physically completely obvious that $\hat{F}$ has an absolutely continuous spectrum. To see this explicitly, suppose that $\eta(\psi)$ is an eigenvector of $\hat{F}$, that is,

$$
\hat{F} \eta(\psi)=\eta(\hat{F} \psi)=\lambda \eta(\psi) .
$$

Since we may assume without loss of generality that $\psi$ depends trivially on $A$, this is equivalent to the equation

$$
\hat{\Pi} \psi=[\lambda+\tau] \psi \Rightarrow \psi(\Phi)=c \exp (-i[\lambda+\tau] \Phi)
$$

for some constant $c$. However, in order to be normalizable in the physical inner product it follows $c=0$. Thus $\hat{F}$ has no pure point spectrum and since the physical Hilbert space is equivalent to $L_{2}(R, d x)$ on which the observable $\hat{F}$ is essentially represented as $i d / d x$ it follows that $\hat{F}$ has an absolutely continuous spectrum as claimed. This example illustrates nicely the heuristic argument given in Sec. II: The flow associated with the partial observable $f=E$ is compact on the kinematical phase space, since it integrates to circles. Under the projection to the (gauge fixed and) constraint hypersurface the flow is mapped to noncompact spirals, leading to a continuous spectrum of the associated Dirac observable.

\footnotetext{
${ }^{1}$ A. Ashtekar, "An introduction to loop quantum gravity through cosmology," Nuovo Cimento B 122, 135 (2007); A. Ashtekar and J. Lewandowski, Class. Quantum Grav. 21, R53 (2004), e-print arXiv:gr-qc/0404018; A. Perez, "Introduction to loop quantum gravity and spin foams," e-print arXiv:gr-qc/0409061 ; C. Rovelli, Quantum Gravity (Cambridge University Press, Cambridge, 2004); L. Smolin, "How far are we from the quantum theory of gravity?," e-print arXiv:hep-th/0303185 ; L. Smolin, "An invitation to loop quantum gravity," Lect. Notes Phys. 721, 185 (2007).; T. Thiemann, "Lectures on loop quantum gravity," Lect. Notes Phys. 631, 41 (2003); T. Thiemann, Lect. Notes Phys. 631, 41 (2003), e-print arXiv:gr-qc/0210094; T. Thiemann, Introduction to Modern Canonical Quantum General Relativity
}

${ }^{6}$ The rigging map maps kinematical wave functions to elements of the physical Hilbert space and is used to define the physical inner product. For more details see, for instance, the last reference of Ref. 1. 
(Cambridge University Press, Cambridge, 2007); e-print arXiv:gr-qc/0110034.

${ }^{2}$ C. Rovelli and L. Smolin, Nucl. Phys. B 442, 593 (1995); C. Rovelli and L. Smolin, ibid. 456, 753(E) (1995), e-print arXiv:gr-qc/9411005.

${ }^{3}$ A. Ashtekar and J. Lewandowski, Class. Quantum Grav. 14, A55 (1997), e-print arXiv:gr-qc/9602046; A. Ashtekar and J. Lewandowski, Adv. Theor. Math. Phys. 1, 388 (1998), e-print arXiv:gr-qc/9711031; A. Ashtekar, A. Corichi, and J. A. Zapata, Class. Quantum Grav. 15, 2955 (1998), e-print arXiv:gr-qc/9806041; T. Thiemann, J. Math. Phys. 39, 3372 (1998), e-print arXiv:gr-qc/9606092.

${ }^{4}$ A. Ashtekar and C. J. Isham, Class. Quantum Grav. 9, 1433 (1992), e-print arXiv:hep-th/9202053; A. Ashtekar and J. Lewandowski, J. Math. Phys. 36, 2170 (1995), e-print arXiv:gr-qc/9411046.

${ }^{5}$ T. Thiemann, J. Math. Phys. 39, 3347 (1998), e-print arXiv:gr-qc/9606091; R. Loll, Nucl. Phys. B 500, 405 (1997), e-print arXiv:gr-qc/9706038; J. Brunnemann and T. Thiemann, Class. Quantum Grav. 23, 1289 (2006), e-print arXiv:grqc/0405060; J. Brunnemann and D. Rideout, "Spectral analysis of the volume operator in loop quantum gravity," e-print arXiv:gr-qc/0612147 ; J. Brunnemann and D. Rideout, "Properties of the volume operator in loop quantum gravity. I. Results," Class. Quantum Grav. 25, 065001 (2008); J. Brunnemann and D. Rideout, "Properties of the volume operator in loop quantum qravity. II. Detailed presentation," e-print arXiv:gq-qc/0706.0382.

${ }^{6} \mathrm{H}$. Sahlmann, "Some comments on the representation theory of the algebra underlying loop quantum gravity," e-print arXiv:gr-qc/0207111 ; H. Sahlmann, "When do measures on the space of connections support the triad operators of loop quantum gravity?," e-print arXiv:gr-qc/0207112 ; H. Sahlmann and T. Thiemann, "On the superselection theory of the Weyl algebra for diffeomorphism invariant quantum gauge theories," e-print arXiv:gr-qc/0302090; H. Sahlmann and T. Thiemann, Commun. Math. Phys. 285, 67 (2009); C. Fleischhack, "Representations of the Weyl algebra in quantum geometry," e-print arXiv:math-ph/0407006v2 ; J. Lewandowski, A. Okolow, H. Sahlmann, and T. Thiemann, "Uniqueness of diffeomorphism invariant states on holonomy-flux algebras," Commun. Math. Phys. 267, 703 (2006).

${ }^{7}$ T. Thiemann, Phys. Lett. B 380, 257 (1996), e-print arXiv:gr-qc/9606088; T. Thiemann, Class. Quantum Grav. 15, 839 (1998), e-print arXiv:gr-qc/9606089; T. Thiemann, ibid. 15, 875 (1998), e-print arXiv:gr-qc/9606090; T. Thiemann, ibid. 15, 1207 (1998), e-print arXiv:gr-qc/9705017; T. Thiemann, ibid. 15, 1249 (1998), e-print arXiv:gr-qc/9705018; T. Thiemann, ibid. 15, 1281 (1998), e-print arXiv:gr-qc/9705019; T. Thiemann, ibid. 15, 1463 (1998), e-print arXiv:gr-qc/ 9705020.

${ }^{8}$ B. Dittrich, "Partial and complete observables for Hamiltonian constrained systems," Gen. Relativ. Gravit. 39, 1891 (2007); 23, 6155 (2006); B. Dittrich, Class. Quantum Grav. 24, 757 (2007); B. Dittrich and J. Tambornino, ibid. 24, 4543 (2007); e-print arXiv:gr-qc/0610060 ; B. Dittrich and J. Tambornino, "Gauge invariant perturbations around symmetry reduced sectors of general relativity: Applications to cosmology," Class. Quantum Gravit. (to be published); e-print arXiv:gr-qc/0702093.

${ }^{9}$ T. Thiemann, Class. Quantum Grav. 23, 1163 (2006), e-print arXiv:gr-qc/0411031.

${ }^{10}$ C. Rovelli, Phys. Rev. D 43, 442 (1991); C. Rovelli, in Conceptional Problems in Quantum Gravity, edited by A. Ashtekar and J. Stachel (Birkhäuser, Boston, 1991), p. 126; C. Rovelli, Class. Quantum Grav. 8, 297 (1991); C. Rovelli, Phys. Rev. D 65, 124013 (2002), e-print arXiv:gr-qc/0110035.

${ }^{11}$ L. Freidel, E. R. Livine, and C. Rovelli, Class. Quantum Grav. 20, 1463 (2003), e-print arXiv:gr-qc/0212077.

${ }^{12}$ K. Noui and A. Perez, Class. Quantum Grav. 22, 4489 (2005), e-print arXiv:gr-qc/0402111.

${ }^{13}$ S. Alexandrov and Z. Kadar, Class. Quantum Grav. 22, 3491 (2005), e-print arXiv:gr-qc/0501093.

${ }^{14}$ A. Messiah, Quantum Mechanics (North-Holland, Amsterdam, 1961), Vol. 1.

${ }^{15}$ R. Brunetti and K. Fredenhagen, "When does a detector click?," e-print arXiv:quant-ph/0103144 ; R. Brunetti and K. Fredenhagen, "Remarks on time-energy uncertainty relations," Rev. Math. Phys. 14, 897 (2002); e-print arXiv:quant-ph/0207048 ; K. Fredenhagen, in Quantum Theory and Symmetries IV, edited by V. K. Dobrev (Heron, Sofia, 2006).

${ }^{16}$ D. Basu and K. W. Wolf, J. Math. Phys. 24, 478 (1983).

${ }^{17}$ R. Gambini and R. A. Porto, Phys. Rev. D 63, 105014 (2001),e-print arXiv:gr-qc/0101057. 